\title{
The study of the on-line fault diagnosis method for induction motor bearing based on AR Model Parameters Identification
}

\author{
YUAN Ju-mei ${ }^{1, \text { a }}$,ZHAO Lu ${ }^{2, b}$ \\ ${ }^{1}$ Department of Automation, Taiyuan Institute of Technology, Taiyuan, 030008, China \\ ${ }^{2}$ Space Science and Technology Institute of Southern China, Shenzhen, 518108, China \\ aemail:yuanjm2005\%126.com, ${ }^{b}$ email:zhaol@126.com
}

Keywords: AR model parameters; Identification; Motor bearing fault; On-line diagnosis

\begin{abstract}
To realize the online fault diagnosis for induction motor bearings, a method of online identification based on the AR model parameter recursive identification for induction motors vibration signal was proposed. Firstly, based on the optimal instrumental variable method, four auto-regression models were established for the vibration signals of induction motor under four conditions: the normal condition, out-race fault, inner-race fault and the ball bearing fault. Then, the state equations for the induction motor vibration signals were established by taking the autoregressive model coefficients as the state variables and the AR model coefficients in normal conditions are initial values. The online parameter identification is finished with Kalman filtering technique. Based on these, the relationship of the model coefficients under the four conditions was analyzed by making use of Hierarchical Cluster Analysis, and the online diagnosis for bearing faults in induction motors was accomplished with the Bullock distance of model parameters as criterion. Finally, the feasibility and the effectiveness of the proposed method are proved through the analysis of examples.
\end{abstract}

\section{Introduction}

As one of the important electric equipments, induction motors are widely used in industrial and agricultural production and daily life. Bearing is an important component to ensure the motors operating normally, but the damage of it takes about $40 \%$ of the total faults of induction motor, which is one of the easiest to failure components in a motor. Correct fault diagnosing for bearing has a great practical significance for prolong the ser-vice life of the motor and save the use cost.

According to different adopted state variables, the induction motor bearing fault detection and diagnosis can be classified as the oil sample analysis method, the temperature method, the vibration signal analysis method and the stator current analysis method [1, 2]. The vibration signal analysis method is one of the best practical diagnostic methods currently, which can detect all types of fault information. The traditional processing methods of vibration signals are to analyze measured signals directly with correlation function, sufficient statistics, time domain index, frequency domain index etc, and to extract time domain and frequency domain feature values such as variance, kurtosis, and the center of power spectrum to realize the faults detection. Recently, high order spectrum, wavelet transform, fractal method, the absolute value of test and the trend test, information fusion, Kullback information criterion method have emerged[3]. These methods belong to off-line fault diagnosis method and it couldn't realize fault detection and diagnosis online. In recent years, the vibration signal modeling method based on time series analysis arouses the attention of researchers. This method uses the actual test data for modeling directly. The time series spectrum is dynamic spectrum that it can extension the observation data, so it has advantages of no energy leakage and high resolution. It overcomes defects as spectral line leakage (power leakage), side lobe appearance, low resolution and signal submerging caused by frequency truncation in the application of Fourier spectral analysis $[4,5]$. According to the reference materials, these methods are based on a once complete algorithm, which occupies too much memory space. Besides, this algorithm is difficult to track the vibration signal change process when the working condition changes. To avoid these drawbacks, an 
induction motor vibration signal identification method based on recursive parameter model identification is presented. This method not only takes up less memory but also can carry out motor bearing fault detection and diagnosis on-line according to the parameters variations. The correctness and validity were proved through the analysis of examples.

\section{The Time Series Model Establishment for Induction Motor Vibration Signal}

In order to study the change laws of time series, its mathematical model is established, which are collectively called the time series model. Time series model can be expressed as three forms: auto-regression model AR (m), moving average model MA (n) and autoregressive moving average model ARMA (m, n).

Setting up a time series model needs to first determine which model the sequence belongs to. If the autocorrelation function $\hat{\rho}(r)$ of the data is truncated when $r>n$, the sequence $\left\{y_{t}\right\}$ is an MA (n) sequence. If the sample partial autocorrelation function $\hat{\phi}(r)$ is truncated when $r>m$, the sequence $\left\{y_{t}\right\}$ is an AR (m) sequence. While the autocorrelation function $\hat{\rho}(r)$ and partial autocorrelation function $\hat{\phi}(r)$ of the data are not truncated, and they are controlled by negative exponential function (trailing), it should be an ARMA model[6].

We use the motor bearings test data provided by the Case Western Reserve University Bearing Data Center Website[7]. Experiments were conducted using a 2 hp Reliance Electric motor, and acceleration data was measured at locations near to and remote from the motor bearings. We select drive end bearing to analysis. This is 6205-2RS JEM SKF, deep groove ball bearing, Faults ranging select 0.007 inches in diameter at the outer raceway; inner raceway and rolling element (i.e. ball). Faulted bearings were reinstalled into the test motor and vibration data was recorded for motor loads of 0 horsepower (motor speeds of 1797 RPM). Data was collected for normal bearings, single-point drive end defects. Data was collected at $12,000 \mathrm{~Hz}$ for drive end bearing experiments. These autocorrelation and partial autocorrelation were analysis for four cases and were shown in figure 1.

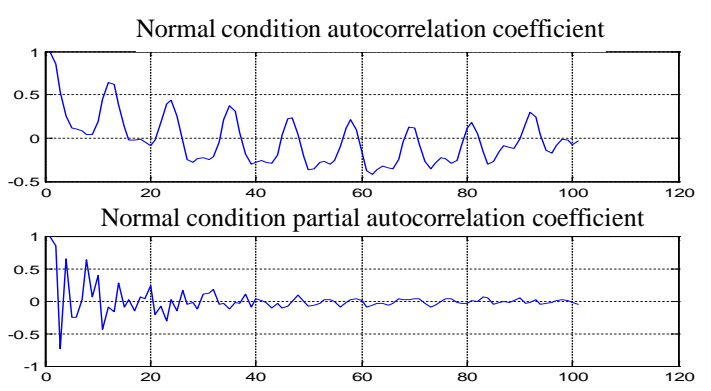

a) Normal condition autocorrelation and partial autocorrelation function Inner raceway fault autocorrelation coefficient
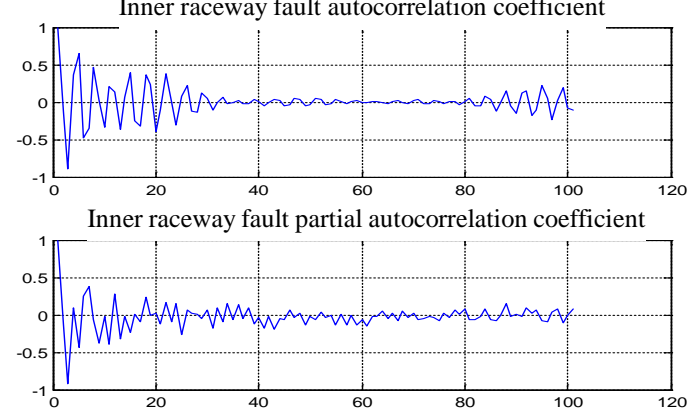

C) Inner raceway fault autocorrelation and partial autocorrelation function

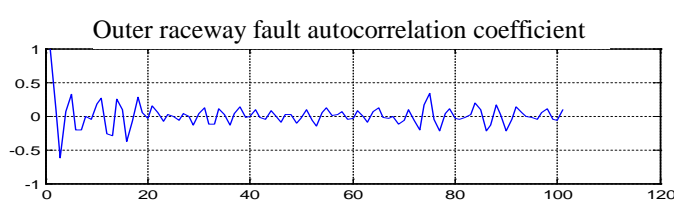

Outer raceway fault partial autocorrelation coefficient

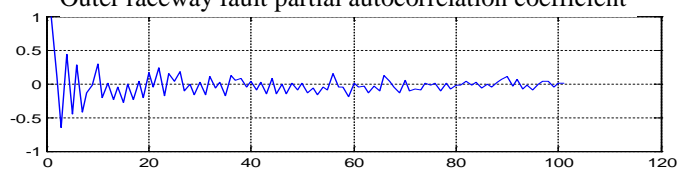

b) Outer raceway fault autocorrelation and nartial autocorrelation function
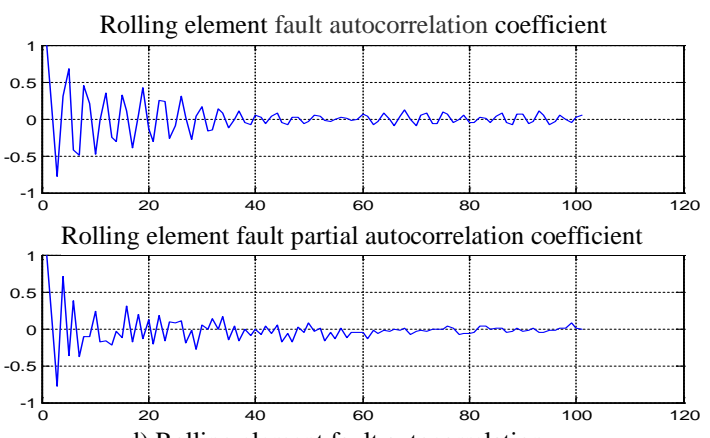

d) Rolling element fault autocorrelation and nartial autocorrelation function

Fig.1 Four conditions autocorrelation and partial autocorrelation function 
From Figure 1 it can be seen that the autocorrelation functions are trailing under four conditions and the partial correlation function have relatively good convergence. They are relatively small when $r>40$ and can be regarded as censored in 40 . As a result, the AR model is selected for motor vibration signal timing model, and the order is 40 .

The methods to estimate the model parameters have the forward and backward method, the least square method, the Yule-Walker method and the instrumental variable method. Here the instrumental variable method is used to determine the model parameters to solve the problems of unbiasedness and consistency of parameters estimation. The time series modeling are established measured vibration acceleration signal under four conditions. The model forms are shown as formula 1.

$$
A(q) y(t)=e(t)
$$

Under the normal condition,

$$
\begin{aligned}
& \mathrm{A}(\mathrm{q})=1-2.204 \mathrm{q}^{-1}+2.326 \mathrm{q}^{-2}-2.166 \mathrm{q}^{-3}+2.536 \mathrm{q}^{-4}-3.121 \mathrm{q}^{-5}+3.716 \mathrm{q}^{-6}-4.316 \mathrm{q}^{-7} \\
& +4.63 \mathrm{q}^{-8}-4.688 \mathrm{q}^{-9}+4.701 \mathrm{q}^{-10}-4.965 \mathrm{q}^{-11}+5.161 \mathrm{q}^{-12}-5.106 \mathrm{q}^{-13}+5.023 \mathrm{q}^{-14} \\
& -4.994 \mathrm{q}^{-15}+4.903 \mathrm{q}^{-16}-4.837 \mathrm{q}^{-17}+4.781 \mathrm{q}^{-18}-4.511 \mathrm{q}^{-19}+4.16 \mathrm{q}^{-20}-3.926 \mathrm{q}^{-21} \\
& +3.633 \mathrm{q}^{-22}-3.301 \mathrm{q}^{-23}+3.015 \mathrm{q}^{-24}-2.593 \mathrm{q}^{-25}+2.336 \mathrm{q}^{-26}-2.186 \mathrm{q}^{-27}+1.881 \mathrm{q}^{-28} \\
& -1.635 \mathrm{q}^{-29}+1.574 \mathrm{q}^{-30}-1.329 \mathrm{q}^{-31}+0.9912 \mathrm{q}^{-32}-0.8397 \mathrm{q}^{-33}+0.6477 \mathrm{q}^{-34}-0.523 \mathrm{q}^{-35} \\
& +0.4981 \mathrm{q}^{-36}-0.3446 \mathrm{q}^{-37}+0.1324 \mathrm{q}^{-38}-0.005906 \mathrm{q}^{-39}-0.01451 \mathrm{q}^{-40}
\end{aligned}
$$

Similarly, the AR models of the vibration signal for out-race fault, inner-race fault and ball bearing faults can be established.

\section{Recursive Identification for Model Parameters}

Recursive identification algorithm usually has the form of [8]:

$\hat{\varphi}(t)=\hat{\varphi}(t-1)+K(t)(y(t)-\hat{y}(t))$

In this formula, $\hat{\varphi}(t)$ is the estimation of parameters at the $t$ moment, $y(t)$ is the output measured at the $t$ moment, $\hat{y}(t)$ is the forecast output according to identification model at the same moment, and $K(t)$ is the gain matrix of the identification. Usually, $K(t)$ is defined as $K(t)=M(t) \Phi(t)$.

For linear regression models like the $\mathrm{AR}, \Phi(t)$ is the changing gradient of $\hat{y}(t)$ to the parameters $\varphi$, i.e.

$$
y(t)=\Phi^{T}(t) \varphi_{0}(t)+v(t)
$$

Where $\varphi_{0}(t)$ is the actual parameter vector, $v(t)$ is the noise.

\section{Recursive Identification Algorithm for The Parameters of The Vibration Signal Model of Induction Motor}

Assume that the change of the actual parameter vector of the object in formula (4) satisfies stochastic walking relations, which can be expressed as:

$$
\begin{aligned}
& \varphi_{0}(t)=\varphi_{0}(t-1)+w(t) \\
& E w(t) w^{T}(t)=Q
\end{aligned}
$$

Then the optimal gain matrix $K(t)$ in identification algorithm may be obtained according to the Kalman filtering [9].

We use this method to recursively identify the model parameters based on Kalman filtering for the coefficients in formula (1) of the vibrating signals model under the normal conditions which is established in section 1 . The initial value of the recursive parameter use the coefficient obtained in section 1 as is shown in formula (2). Then the time-domain signal and frequency spectrum of the 
actual measured vibration acceleration under the normal condition, and the predicting values derived from Kalman filter recursive parameters identification are gotten. The results are shown in figure 2.

From Fig. 2 a) and b), we can conclude that the model parameters derived from the identification method of recursive parameters model based on Kalman filter can accurately track the actual measured vibration signals of motor. Figure 2 c) and d) present that the predicted value after recursive parameter identification has good filter effect for actual measurement signal, and it retains the information of the characteristic frequency of original signals.

The time-domain signal and frequency spectrum of the measured vibration acceleration signals and the predicting values derived from Kalman filter recursive parameters identification under out-race fault, inner-race fault and ball bearing fault conditions can be obtained by using the same method.
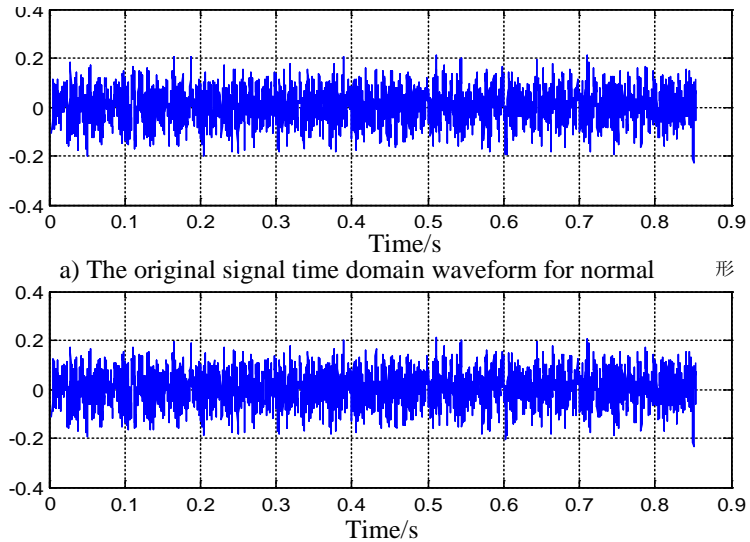

b) After filter time domain waveform for normal
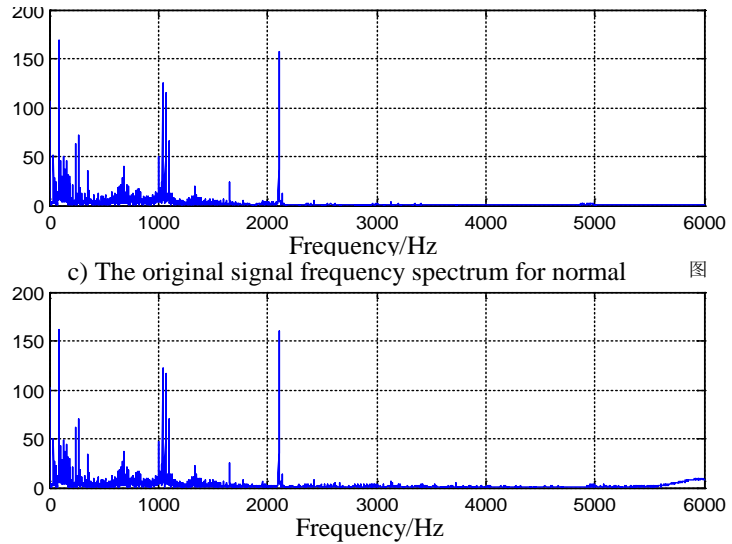

d) After filter frequency spectrum for normal

Fig.2 Time domain and frequency spectrum of before and after filtering for normal conditions

Assume that the out-race fault occurred after the motor runs 0.42675 seconds. The measured vibration acceleration time domain signal is shown in Figure 3. The predicted values after Kalman filter recursive parameters identification are shown in Figure 4, and the residual signals are shown in Figure 5.

The predicted values obtained with non-recursive parameter modeling identification are shown in Figure 6 and the residual signals are shown in Figure 7.

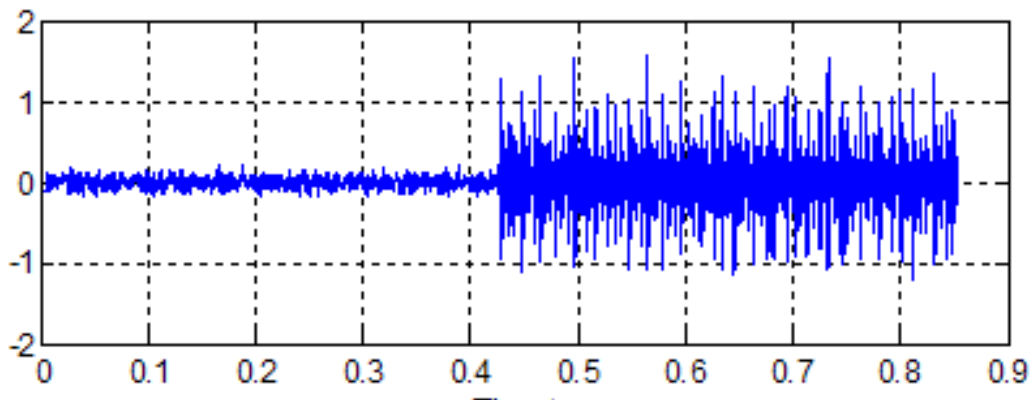

Fig.3 The actual signal of outer raceway fault occurred after normal a period

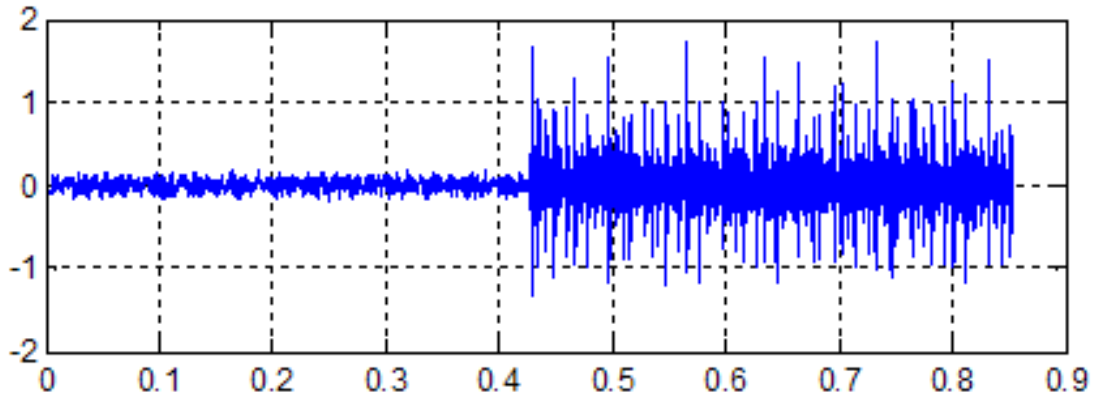




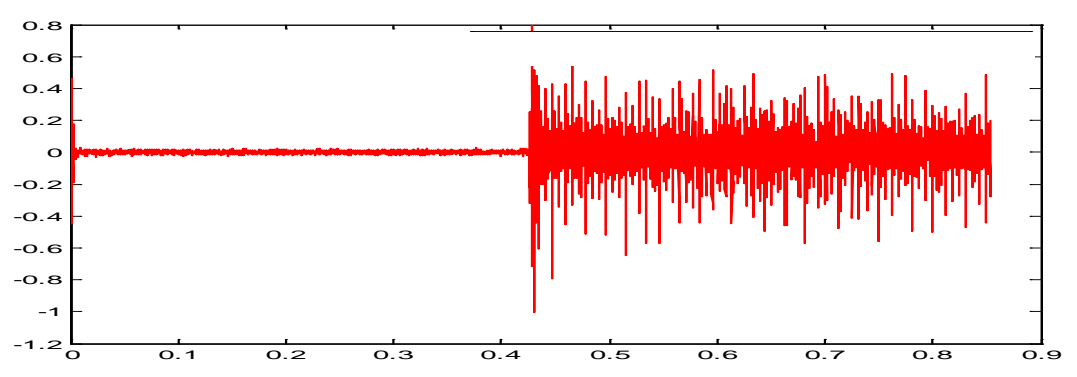

Fig.5 Residual of recursive predictive values for outer raceway fault after normal a period

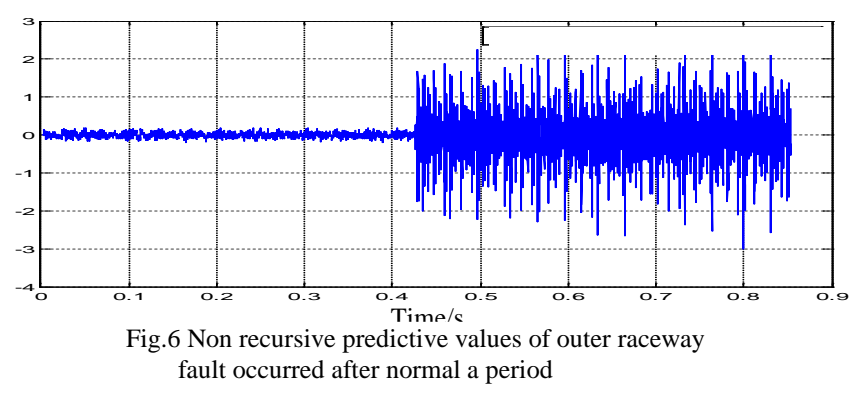

Fig.4 Recursive predictive values of outer raceway fault occurred after normal a period

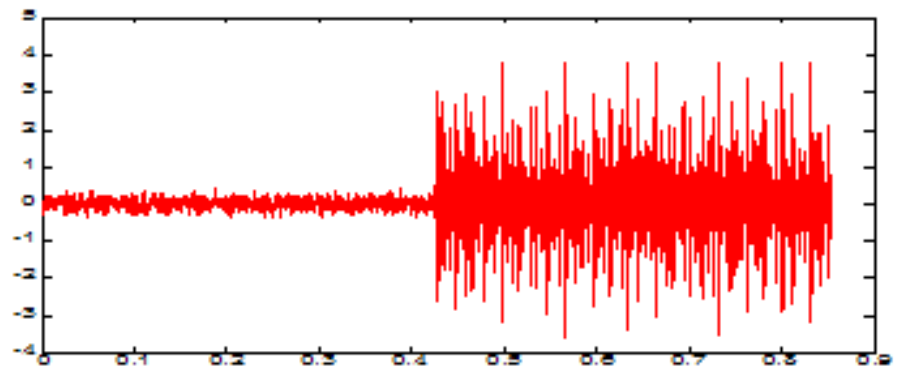

Fig.7 Residual of non recursive predictive values for outer raceway fault after normal a period

Compare the Figure 4 with Figure 6 and Figure 3 , we can decide that the tracking effect of the prediction values to measured signals using AR model parameter recursive identification algorithm is obviously better than non-recursive parameter identification algorithm. As it can be seen in Figure 5 and Figure 7, the residual signals obtained from non-recursive parameter algorithm are at the same order of magnitude as the real vibration signals, and some value is larger than measured value, but the residual signals gotten from the recursive parameter method is relatively one order smaller than the measured value.

\section{Motor Bearing Online Fault Diagnosis Based on Parameter Identification of AR Model}

With the analyses above, we can know that if the state of the motor maintains the normal working condition, the AR model parameters change in a little scope. However, once the working condition of the motor changes, the model parameters would change inevitable. Therefore, the motor fault can be detected and diagnosed through constructing statistical features of the model parameters. If the constructed statistical characteristic quantity is in a certain range, the state of the motor is considered as having no changes, while if this statistics exceeds a certain range, the status of the motor is considered as in malfunction.

The vibration signals for four conditions were sampled eight times respectively, and the AR models were established for each signal. In total, 32 AR models for vibration signals were obtained.

These model parameters are analyzed by K-means clustering. The classification distance adopt Squared Euclidean distance, Sum of absolute differences, a.k.a. L1 distance, One minus the cosine of the included angle between points (treated as vectors) and One minus the sample correlation between points (treated as sequences of values) respectively. The analysis results are shown in Table 1.

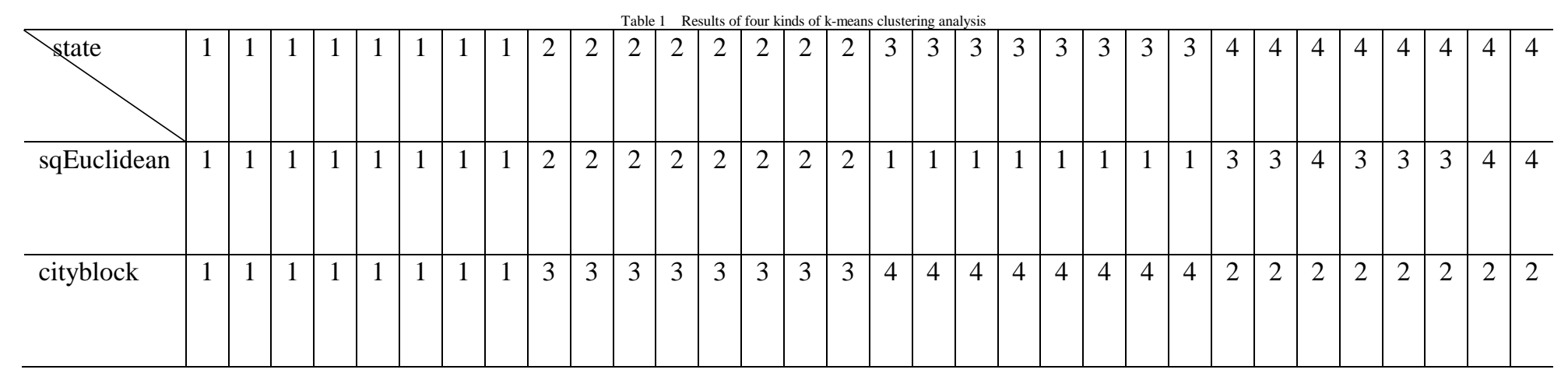




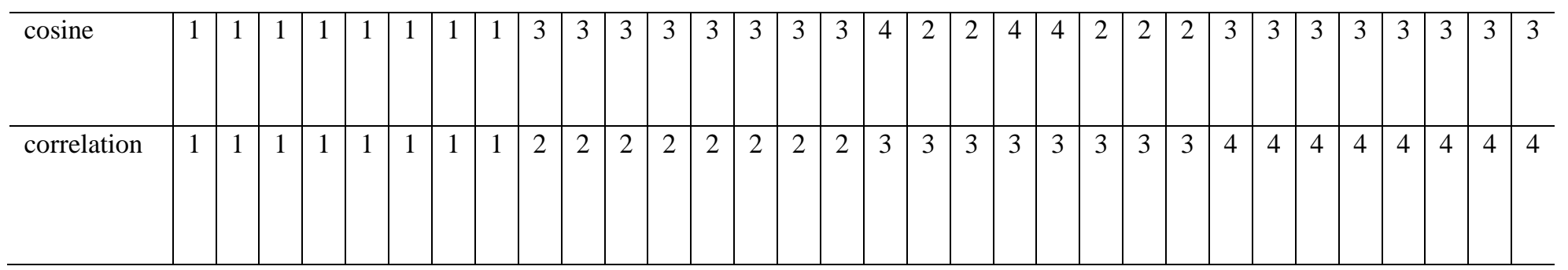

From Table 1, we can acquire that Sum of absolute differences, a.k.a. L1 distance(Block distance) and One minus the sample correlation between points can correctly classify the four working states. The Squared Euclidean distance and one minus the cosine of the included angle between points cannot categorize four working states correctly and the error rate is $34 \%$. Therefore, the sum of absolute differences, a.k.a. L1 distances are selected as the statistics of the model parameters, meanwhile, the clustering centers of four states are determined.

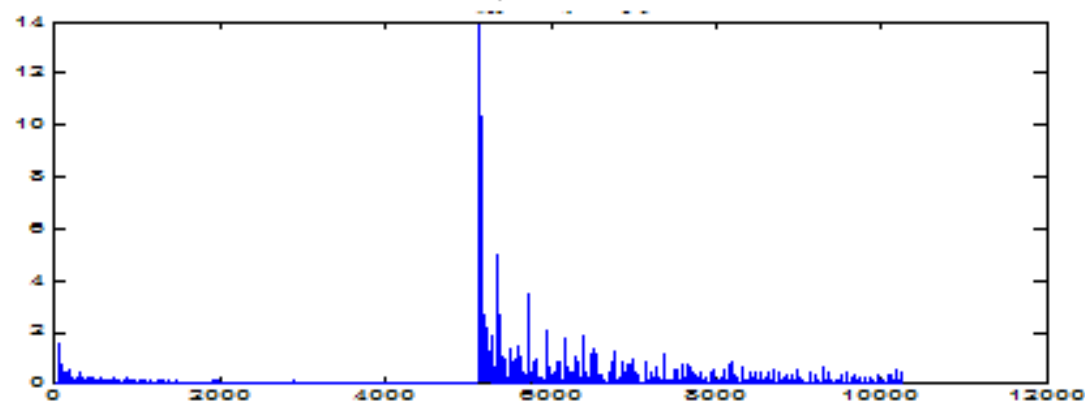

Fig.8 Block distance of adjacent parameters for outer raceway fault occurred after normal a period

We calculate the Block distance of adjacent parameters of the recursive model parameters obtained from above which occurring the out-race fault after normal running for a period. It is showed in Fig.8.

In the same way, we can obtain the Block distance of adjacent parameters of the recursive model parameters which occurring inner-race fault and the ball bearing fault after normal running for a period.

As is shown in Figure 8, the Block distance of adjacent parameters fluctuated slightly when motor kept a normal work state. However, when out-race fault suddenly appeared, the Block distance of adjacent parameters would change dramatically, and then the motor would be in an out-race fault state. After handled with the recursive parameter model identification, the Block distance of adjacent parameters will be small again. Because the fault generated at 0.42675 second after the system run normally, i.e. it happened at $1521^{\text {st }}$ sampling point. The maximum Block distance of the adjacent recursive parameter appears in No. 5140 sampling point i.e. $0.4283 \mathrm{~s}$. The number of the motor sampling points is 400 each turn, which means that the motor fault can be detected after it turns about 0.05 circles under the faulty condition.

When the occurring fault is detected, the distances between the coefficients of fault point model and the cluster centers under four conditions should be calculated. The class with the minimum distance is the class of the motor status belongs to. In this paper, the calculated distances between the model coefficients of the $5140^{\text {th }}$ point and the cluster center of four states respectively are 95.585, $26.868,65.709$ and 29.754. The second category whose distance is the smallest belongs to the class of out-race fault.

The ball bearing fault can be diagnosed in the same way. Assume that the ball bearing fault happened after the motor ran 0.42675 seconds in a normal state which the sampling point is $5121^{\text {st }}$.The maximum Block distance of the adjacent recursive parameters appeared in $5138^{\text {th }}$ sampling point, the time is at the $0.4282 \mathrm{~s}$. The distances of the model coefficients at the time and the cluster center of four states are respectively 63.692, 49.775, 34.390 and 4.0899. The fourth category 
with the minimum distance is the class of ball bearing fault. It can be concluded that this approach is available for diagnosing the faults of induction motors online.

\section{Conclusions and Future Work}

On the basis of timing model establishment for the vibration signals of the induction motor bearing under four conditions, we studied the recursive parameter model identification technology, and applied the Kalman filtering technique to the recursive identification algorithm for the parameters of the vibration signal model of induction motor. Meanwhile, we made the K-means clustering analysis for the timing model parameters under four conditions. It shows that the Block distance of model parameters can be seen as the criterion of the online fault diagnosis for induction motor bearing. It has been illustrated with a case that the fault occurrence and the fault type of induction motor can be detected in 0.05 turning circle time after the out-race fault occurs, which proves the feasibility and effectiveness of the proposed method. Further research work is still needed to improve the recursive parameter identification method to find faults more rapidly.

\section{Acknowledgement}

This work is supported by the Natural Science Foundation of Shanxi Province(Project No.2013011018-2). The authors would like to express their gratitude for the support of this study

\section{References}

[1] R.R.SCHOEN,T.G HABETLER,F. KAMRAN, et a1.Motor bearing damage detection using stator current monitoring. IEEE Trans. on Industry Applications,1995,31(6):1274 1279.

[2] Liu Shaofeng. The study of induction motor fault detection method based on electrical and mechanical signals[D]. North China Electric Power University.2008

[3] Chen YuDong, Shi Songjiao, Weng zhengxin. Review of fault diagnosis method for dynamic system [J]. Chemical Automation and Instrumentation,2001,28(3): 9 10.

[4]Yao zhuting, Pan hongxia. Modeling and Identification for armored vehicle gear main transmission system [J]. Vibration, Test and Diagnostics. 2005,25(3):196 199

[5] Huang guolong. The study of rotating machinery fault diagnosis and status prediction based on order tracking and AR Model[D]. Tianjin University.2008.06

[6] He shuyuan. Application of Time Series Analysis [M].Peking University Press.2003.09

[7]Case Western Reserve University. Bearing Date Center Seeded Fault Test Date [DB]. Cleveland(USA),2008

[8]Li yanjun, Zhang ke and etc. System Identification Theory and Application [M], Defense Industry Press.2003.01

[9]Liu bao, Tang wansheng and etc. Modern Control Theory [M], Machinery Industry Press, 2006.09 